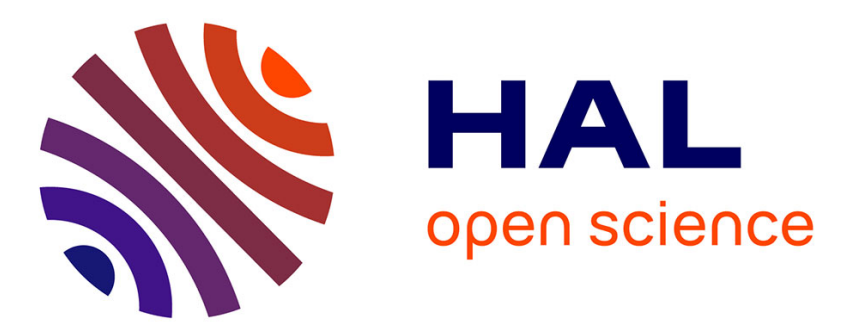

\title{
Computing cross-sections of the workspace of suspended cable-driven parallel robot with sagging cables having tension limitations
}

Jean-Pierre Merlet

\section{- To cite this version:}

Jean-Pierre Merlet. Computing cross-sections of the workspace of suspended cable-driven parallel robot with sagging cables having tension limitations. IROS, 2018, Madrid, Unknown Region. hal01965229

\section{HAL Id: hal-01965229 \\ https://hal.science/hal-01965229}

Submitted on 25 Dec 2018

HAL is a multi-disciplinary open access archive for the deposit and dissemination of scientific research documents, whether they are published or not. The documents may come from teaching and research institutions in France or abroad, or from public or private research centers.
L'archive ouverte pluridisciplinaire HAL, est destinée au dépôt et à la diffusion de documents scientifiques de niveau recherche, publiés ou non, émanant des établissements d'enseignement et de recherche français ou étrangers, des laboratoires publics ou privés. 


\title{
Computing cross-sections of the workspace of suspended cable-driven parallel robot with sagging cables having tension limitations
}

\author{
J-P. Merlet ${ }^{1}$
}

\begin{abstract}
Although workspace is essential for the design and control of cable-driven parallel robots (CDPR) very few works have been devoted to this topic when sagging cables are considered, most probably because of the complexity of the cable model. In this paper we consider suspended CDPR with sagging cables that can support only a limited tension. We propose an algorithm to compute the border of horizontal cross-sections of the workspace for a given altitude and orientation of the platform. We show that singularities of the kinematics equations have to be taken into account for a proper determination of the border and that the workspace can be separated in several components according to the branch of the inverse kinematics on which the robot is evolving. We also compare the workspace obtained for ideal and sagging cables.
\end{abstract}

\section{INTRODUCTION}

Workspace is an essential element for the design and control of a robot. As far as parallel robots with rigid legs are concerned efficient algorithms have been proposed to compute the border of cross-section of the workspace when the orientation and altitude are fixed, taking into account various limitations such as constraints on the leg lengths, on the amplitude of the passive joint motion or interference between the legs [1] which are basically geometrical limitations. We consider here a variant of parallel robots, namely cable-driven parallel robots (CDPR) in which the rigid legs of parallel robots are substituted by coilable/uncoilable cables. For CDPR any kinematic problem involves also cable tensions as cable can exert only a pulling action so that the name kineto-static is often used. The complexity of managing cable tensions is highly dependent upon the cable modeling. In this paper we are interested in workspace calculation when the cable tensions shall be lower than a given threshold $T$ and the platform is submitted to a constant wrench $\mathcal{F}$ (e.g gravity). If the CDPR cables are assumed to be ideal (i.e. no deformation due to the weight of the cable, no elasticity) the mechanical equilibrium constraint establishes a linear relationship between the tension $\tau$ in the cables and $\mathcal{F}$ that is written as $\mathcal{F}=\mathbf{J}^{-\mathbf{T}}(\mathbf{X}) \tau$ where $\mathbf{J}^{-\mathbf{T}}$ is the transpose of the inverse kinematic jacobian and $\mathbf{X}$ is the pose of the CDPR. The workspace is the region of the operational space such this relation is satisfied with all components $\tau_{i}$ of $\tau$ satisfying $0 \leq \tau_{i} \leq T$ (the positiveness of $\tau_{i}$ being imposed because cable cannot push). Several papers have addressed workspace calculation in that case [2], [3], [4],[5], [6], [7],[8], [9], [10], [11],[12], [13], [14]. Workspace analysis for CDPR with elastic cable but without shape deformation has been less considered [15],

\footnotetext{
${ }^{1}$ HEPHAISTOS project, Université Côte d'Azur, Inria, France Jean-Pierre.Merleteinria.fr
}

[16]. If sagging cables are assumed, as this will be the case in this paper, the problem becomes more complex and to the best of the author's knowledge this problem has only been addressed in [17]. But the discretisation approach used in this paper is not efficient, especially for large robot, and several theoretical issues have not been addressed. We have proposed a preliminary analysis of this problem for 6 d.o.f robot [18] with the purpose of showing that calculating workspace slices may lead to complex workspace with a large number of disconnected components but the proposed algorithm was computer intensive and was also relying on discretisation.

In this paper we are interested in calculating cross-sections of the workspace of a CDPR for given altitude and orientation of the platform under the constraint that the tension of any cable does not exceed a given threshold. Our objective is to calculate a polygonal approximation of the border of the workspace that can be refined at will.

\section{KinEMATICS}

In this paper we will use the Irvine sagging cable model that is valid for elastic and deformable cable with mass [19] and that has been shown to be in very good agreement with experimental result [20]. This model is established in the cable vertical plane in which we have the upper attachment point of the cable denoted by $A_{i}$ with coordinates $(0,0)$ and the cable lower attachment point denoted by $B_{i}$ with coordinates $\left(x_{b} \geq 0, z_{b}<0\right)$ Vertical and horizontal forces $F_{z}, F_{x}>0$ are exerted on the cable at point $B_{i}$. For a cable with length at rest $L_{0}$ the coordinates of $B$ are given by [19]:

$$
\begin{aligned}
& x_{b}=F_{x}\left(\frac{L_{0}}{E A_{0}}+\frac{\sinh ^{-1}\left(F_{z}\right)-\sinh ^{-1}\left(F_{z}-\frac{\mu g L_{0}}{F_{x}}\right)}{\mu g}\right) \\
& z_{b}=\frac{F_{z}}{E A_{0}}-\frac{\mu g L_{0}^{2}}{2}+ \\
& \frac{\sqrt{F_{x}^{2}+F_{z}^{2}}-\sqrt{F_{x}^{2}+\left(F_{z}-\mu g L_{0}\right)^{2}}}{\mu g}
\end{aligned}
$$

where $E$ is the Young modulus, $A_{0}$ the cable cross-section area, $L_{0}$ the cable length at rest and $\mu$ the cable linear density. We consider a CDPR with $n \geq 6$ cables which is suspended i.e. the winches output point $A_{i}$ is always higher than the attachment point $B_{i}$ of the cable on the platform. Consider a reference frame with a vertical $\mathbf{z}$ axis and the cable frame attached to the cable plane and the rotation matrix between these two frames, that correspond to a rotation around the $\mathbf{z}$ axis of angle $\alpha_{i}$. If the components of vector $\mathbf{A}_{\mathbf{i}} \mathbf{B}_{\mathbf{i}}$ in the reference frame are $x_{r}^{i}, y_{r}^{i}, z_{r}^{i}$, then $\alpha_{i}$ must satisfy the 
equation:

$$
x_{r}^{i} \sin \left(\alpha_{i}\right)+y_{r}^{i} \cos \left(\alpha_{i}\right)=0
$$

If $\left(F_{x}^{i}>0,0, F_{z}^{i}\right)$ are the components of the force that is applied by the platform on the cable at $B_{i}$ in its plane, then the force exerted by the cable on the platform in the reference frame is $\mathbf{F}_{\mathbf{a}}^{\mathbf{i}}=\left(-F_{x}^{i} \cos \left(\alpha_{i}\right),-F_{x}^{i} \sin \left(\alpha_{i}\right),-F_{z}^{i}\right)$. We assume that the platform is submitted only to gravity which exert a vertical force $\mathcal{F}$ and no torque around the platform center of mass $C$. Hence the mechanical equilibrium imposes

$$
\sum_{j=1}^{j=n} \mathbf{F}_{\mathbf{a}}^{\mathbf{j}}+\mathcal{F}=\mathbf{0} \quad \sum_{j=1}^{j=n} \mathbf{C B}_{\mathbf{i}} \times \mathbf{F}_{\mathbf{a}}^{\mathbf{j}}=\mathbf{0}
$$

Kinematic analysis will be based on the $2 n$ equations (1), the $n$ equations (2) and the 6 equations (3) for a total of $3 n+6$ constraints (but a subset of these equations may have to be considered according to the problem). The unknowns will be a subset of the $4 n+6$ variables $F_{x}^{i}, F_{z}^{i}, L_{0}^{i}, \alpha_{i}$ and the pose $\mathbf{X}$ parameters. For example if we are interested in the inverse kinematics problem (IK) the 6 pose parameters and the $n \alpha_{i}$ will be known while the $3 n F_{x}^{i}, F_{z}^{i}, L_{0}^{i}$ will have to be determined and we will have $2 n+6$ constraints. Note that if $n=6$ the IK equations is a square system and it is known that this system may have multiple solution [21]. On the other hand if we are interested in the forward kinematics (FK) we will have $3 n+6$ unknowns $\left(3 n F_{x}^{i}, F_{z}^{i}, \alpha_{i}\right.$ plus the pose parameters) and $3 n+6$ constraints (and consequently the FK is always a square system).

\section{WORKSPACE FOR CDPR WITH 6 CABLES}

To illustrate the border computation we start with a CDPR having 6 cables so that the IK is a square system. As stated in the introduction we will impose a constraint on the cable tensions as $F_{x}^{2}+F_{z}^{2} \leq T^{2}$ where $T$ is the tension threshold. Note that $\sqrt{F_{x}^{2}+F_{z}^{2}}$ is not exactly the maximal tension exerted on a cable, which is $\sqrt{F_{x}^{2}+\left(F_{z}+\mu g L_{0}\right)^{2}}$, but we will assume that $T$ is high enough so that we can neglect the contribution of $\mu g L_{0}$.

As we have assumed that the altitude and the orientation of the platform are fixed we are interested in the possible location of $C$ in an horizontal plane and the position of $C$ in this plane will be denoted by $x, y$. If we let $x, y$ be free the kinematics equations have 26 unknowns $(x, y$ and the $24 F_{x}, F_{z}, \alpha, L_{0}$ ) which are constrained by 24 equations and therefore $C$ lies in a 2D region. The border of this region is an 1-dimensional variety that is obtained when we impose that one of the cable $j$ satisfies $H_{j}=\left(F_{x}^{j}\right)^{2}+\left(F_{z}^{j}\right)^{2}=T^{2}$. Unfortunately being given the nature of the FK equations the elimination of $F_{x}^{i}, F_{z}^{i}, \alpha_{i}, L_{0}^{i}$ from the FK equations in order to get an implicit equation $G_{i}(x, y)=0$ that will describe the border seems to be very difficult. We will thus use another approach: we will determine poses on the border and these poses will be used as starting pose for a continuation approach that will determine successive poses on the border in order to obtain a polygonal approximation of the $G_{i}$ curve. Hence the first step of the algorithm is to find poses, denoted extremal poses, that are on the border.

\section{A. Finding extremal poses}

An extremal poses is characterized by the property that its IK solution is such that for at least one cable $j$ we have $H_{j}=T^{2}$. Cable $j$ will be denoted the leading cable for this extremal pose. For finding extremal poses we will start from a pose $\mathbf{X}_{\mathbf{0}}$ with coordinates $\left(x_{0}, y_{0}\right)$, called an initial pose,that is arbitrary and is assumed to lie in the workspace. We will compute all its $m$ IK solution(s) using the method proposed in [21]. We then consider the pose $\mathbf{X}_{1}$ defined by the coordinates $\left(x_{0}+\epsilon, y_{0}\right)$ where $\epsilon$ is a small value that is determined with the Kantorovitch theorem [22] so that the IK system is guaranteed to have a single solution around $\mathbf{X}_{\mathbf{0}}$ that is guaranteed to be obtained by using the NewtonRaphson scheme using as initial guess the IK solution of $\mathrm{X}_{\mathbf{0}}$.

We then check that at $\mathbf{X}_{\mathbf{1}}$ we have $H_{j}<T^{2}$ for all cables $j$. If this is the case we substitute $\mathbf{X}_{\mathbf{0}}$ by $\mathbf{X}_{\mathbf{1}}$ and repeat the process. Two situations may occur during this process:

- Kantorovitch theorem fails to show that the Newton scheme will converge. In that case we divide $\epsilon$ by 2 and repeat the test. However at some point Kantorovitch theorem may still fail although $\epsilon$ is very small, which means that we are close to a singularity. We will see later on that it is necessary to consider such pose.

- the solution for $\mathbf{X}_{\mathbf{1}}$ is such that for one $j$ we have $H_{j}$ that is very close to $T^{2}$. We will assume that $x$ is a free variable and consider the kinematic problem with 25 unknowns $\left(x, F_{x}, F_{z}, L_{0}, \alpha\right)$ and 24 equations to which we add $H_{j}=T^{2}$. This is a square system that is solved using Newton. If the solution is such that $H_{k} \leq T^{2}$ for all $k \neq j$ and $F_{x}>0$ for all cables then we have obtained an extremal pose. This extremal pose lie on a potential arc for the border, denoted $S_{j}^{k}$, that is associated to cable $j$ and to the solution number $k$ of the IK at $\mathbf{X}_{\mathbf{0}}$ that has been used to derive $\mathbf{X}_{\mathbf{1}}$.

This process is repeated by moving from $\mathbf{X}_{\mathbf{0}}$ in the $x-, y+, y-$ directions, leading to a set of extremal poses $\mathbf{X}_{\mathbf{j}}^{\mathbf{e}}, \mathbf{X}_{\mathbf{k}}^{\mathbf{e}}, \ldots$ that belong to different $\operatorname{arcs} S_{j}, S_{k}, \ldots$ We may now present the continuation algorithm that allow us to obtain a polygonal approximation of these arcs.

\section{B. The continuation algorithm}

Let $\mathbf{X}_{1}=\left(x_{l}, y_{l}\right)$ be an extremal pose lying on the curve $S_{l}$, that, for example, has been obtained when moving along the $x+$ direction. We consider a pose $\mathbf{X}_{\mathbf{k}}$ in a small neighborhood of $\mathbf{X}_{1}$, whose $y$ coordinate will be set to $y_{l}-\gamma$, where $\gamma>0$ is very small. We let $x$ be a free variable and consider the kinematic equations to which we add $H_{l}=T^{2}$ so that we have a square system. By using Newton we are able to determine the $x$ coordinate of $\mathbf{X}_{\mathbf{k}}$ so that this pose belongs to $S_{l}$. With $\mathbf{X}_{\mathbf{l}}, \mathbf{X}_{\mathbf{k}}$ on $S_{l}$ we are able to get an approximation of the unit tangent vector $\mathbf{T}_{1}^{\mathrm{k}}$ of $S_{l}$ at $\mathbf{X}_{\mathbf{k}}$. If the largest component $u$ of $\mathbf{T}_{1}^{\mathbf{k}}$ is its $x$ component we define $x_{k+1}=x_{k}+\epsilon u$ while $y_{k+1}$ will be considered a free variable. In the opposite case we use $y_{k+1}=y_{k}+\epsilon u$ and $x$ will be the free variable. The kinematic equations is a square system and 
$\epsilon$ is determined with Kantorovitch theorem so that Newton is able to calculate the value of the free variable. such that $\mathbf{X}_{\mathbf{k}+\mathbf{1}}=\left(x_{k+1}, y_{k+1}\right)$ belongs to $S_{l}$. We check if $\mathbf{X}_{\mathbf{k + 1}}$ is such that $H_{j} \leq T^{2}$ for all $j \in[1,6], j \neq l$ and if $F_{x}^{k}>0$ for all cables. If this is the case we have obtained a new pose on $S_{l}$ and we repeat the process. The continuation process stops in the following cases:

- if $H_{j}, j \neq l$ is close to $T^{2}$ the kinematic equations with free $x, y$ and the constraints $H_{l}=T^{2}, H_{j}=T^{2}$ is a square system and we use Newton with $\mathbf{X}_{\mathbf{k}}$ as initial guess for solving it. If a solution is found we have obtained a special extremal pose, called an endpoint of $S_{l}$, which has the property of having 2 leading cables

- we get a pose $\mathbf{X}_{\mathbf{u}}$ that is very close to a previously determined pose $\mathbf{X}_{\mathbf{v}}$ on $S_{l}$. This means that $S_{l}$ is now a closed loop

- Newton is not able to solve the kinematic system although $\epsilon$ is very small: we are close to a singularity of the kinematic equations and we will manage this case later on.

When the continuation has stopped we will start a new continuation process for $S_{l}$ using $-\mathbf{T}_{\mathbf{l}}^{\mathbf{k}}$ as tangent at $\mathbf{X}_{\mathbf{k}}$ for the continuation direction. This continuation process is repeated for all starting points of all branches.

\section{Example}

We consider as example our large scale robot MARIONET-CRANE [23], probably one of the largest CDPR ever deployed. This robot is a suspended CDPR with 6 cables, whose $A_{i}, B_{i}$ coordinates are given in table I. We

\begin{tabular}{|c|c|c|c|c|c|c|c|}
\hline $\mathrm{X}$ & $\mathrm{y}$ & \multicolumn{2}{|c|}{ Z } & $\mathrm{x}$ & \multicolumn{2}{|c|}{$\mathrm{y}$} & Z \\
\hline-325.9 & -47.5 & \multicolumn{2}{|c|}{882.6} & 942 & \multicolumn{2}{|c|}{-348.2} & 1155.5 \\
\hline 953.8 & 379.7 & \multicolumn{2}{|c|}{1153.3} & 55 & \multicolumn{2}{|c|}{2041.4} & 870.4 \\
\hline-250.5 & 1681.0 & \multicolumn{2}{|c|}{864.9} & -33 & \multicolumn{2}{|c|}{942.1} & 878.8 \\
\hline & $\mathrm{X}$ & $\mathrm{y}$ & Z & $\mathrm{X}$ & $\mathrm{y}$ & Z & \\
\hline & -10 & -93 & -3 & 10 & -93 & -3 & \\
\hline & 27 & 50 & -7 & 27 & 50 & -7 & \\
\hline & -27 & 50 & -7 & -27 & 50 & -7 & \\
\hline
\end{tabular}

TABLE I

Coordinates of the $A_{i}$ And $B_{i}$ POINTS on the BASE And on the PLATFORM (IN CM, BY ROWS)

assume that the platform mass is $100 \mathrm{~kg}, E=10^{9} \mathrm{~N} / \mathrm{m}^{2}$, $\mu=0.79 \mathrm{~kg} / \mathrm{m}$, the cable diameter is $2 \mathrm{~mm}$ and we set $T$ to $505 \mathrm{~N}$. Our purpose is to compute a slice of the workspace for $z=200$ while the orientation of the platform is such that the rotation matrix for the platform is the identity. Using as starting pose $M=(2,8)$ we use the strategy of section (III-A) to find 3 extremal poses with leading cables $4,5,6$. The result of the continuation method based on these 3 extremal poses is shown in figure 1 .

It appears clearly that the result is not satisfactory because the curves do no provide a closed region. We also note that the two curves with leading cable 4 and 5 are intersecting. This shows that the intersection point is the meeting point

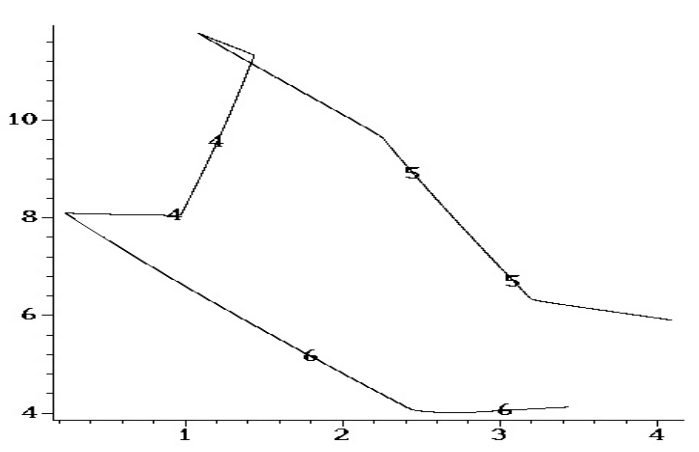

Fig. 1. The border curves derived from the first 3 extremal poses with leading cable $4,5,6$

of 2 kineto-static branches one of which is such that $T_{4}=$ $T, T_{5}<T$ and the other with $T_{4}<T, T_{5}=T$. We will study in depth this case in a later section. For now we will look at the missing border arcs by looking for new extremal pose that will be used as starting point for the continuation algorithm.

To close the region we look at the end-points of the arcs. If such a point is not an end-point of an established curve, then it may be used as a new starting point for the continuation. This process is repeated until all end-points are starting point for the established arcs. In our example figure 2 shows that we obtain now a closed region.

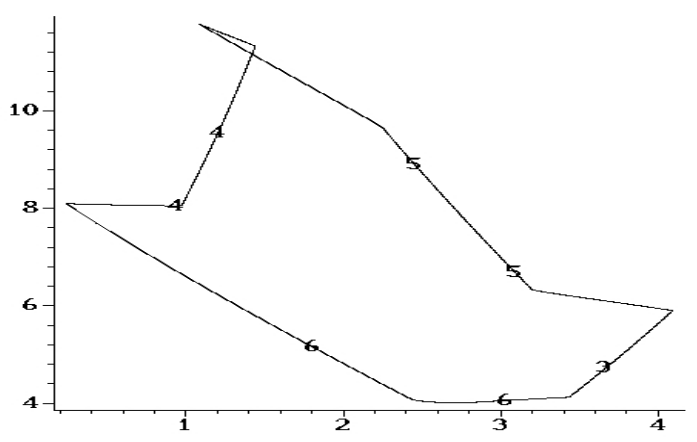

Fig. 2. The border curves after completion by examining the end-points of the arcs

\section{Managing intersection points and singularities}

As seen in the example we may have arcs that intersect. They must lie on different kineto-stack branches of the IK because otherwise these points should have been singular. Let us consider the point $M$ of the example that is the intersection point of $S_{4}, S_{5}$. Starting from this point we will examine what happen when moving in the $x+, x-, y+, y-$ direction. We perform this task using Newton with as starting point the IK solution obtained for $S_{4}, S_{5}$ and we look especially at what happen for $H_{4}, H_{5}$. Figure 3 presents the evolution of $H_{4}, H_{5}$ in both cases. When a motion along 
a given axis leads to a singularity it is denoted $S$. An
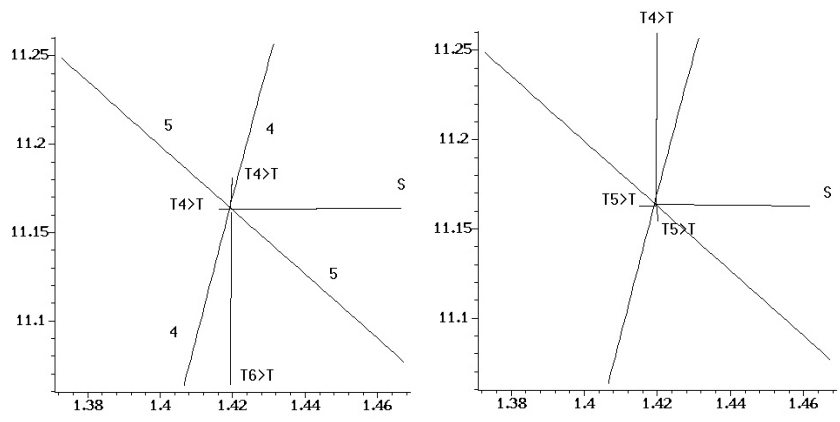

Fig. 3. The evolution of the IK solution from the intersection point when starting with the solution of the IK for $M$ on $S_{4}$ (left) and on $S_{5}$ (right)

interesting point is that when moving from $M$ in the $x+$ direction we get poses for which all $H_{j}$ satisfy $H_{j}<T^{2}$. Thereby there is a region on the right side of $M$ that is included in the workspace although we have not determined any border arc for this region. As a motion in this direction ends up in a singularity there must be clearly a singular arc. Using an adaptation of the continuation algorithm that is not detailed here for lack of space we indeed find a singularity curve $S$ presented in figure 4 . This curve establishes a new

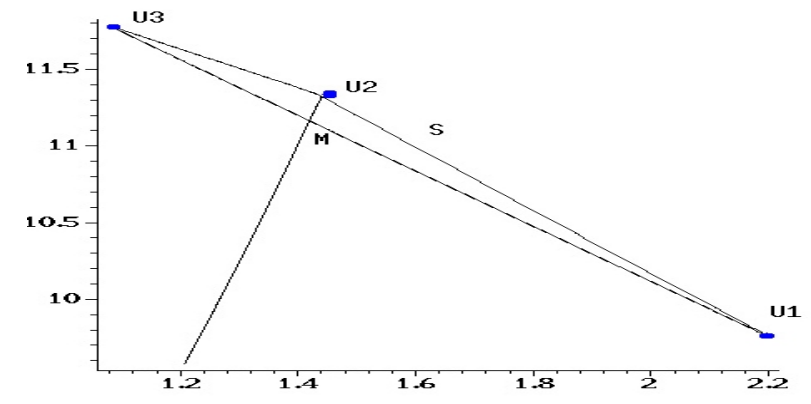

Fig. 4. A detail of the intersection between $C_{4}, C_{5}$ and the new singularity curve (denoted by $\mathrm{S}$ )

component of the border so that the workspace will depend on the kineto-static branches. Figure 5 presents the obtained workspace when the IK solution is on the branch of $S_{4}$ and when it is on the branch of $S_{5}$. A consequence is that the upper left point $U_{3}$ of the workspace cannot be reached using the IK branch of $S_{4}$ : we have first to move in the intersection between the two workspaces (region $M U_{2} U_{1}$ ), apply the IK solution for the $S_{5}$ branch and then move toward $U_{3}$, Hence when computing the workspace it is necessary to check if two $S_{j}, S_{k}$ intersect, which is an easy task. Then an analysis of the evolution of $H_{j}, H_{k}$ around the intersection point is required.

\section{E. Managing extremal cable length}

Some of the IK solution obtained for CDPR may have very large cable lengths (several hundred meters for our example), leading to unrealistic configuration. A first possibility to manage this situation is to impose that the lowest point of a
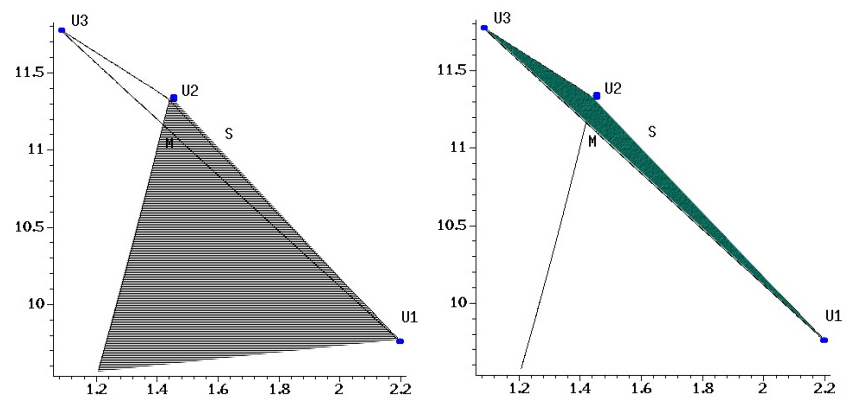

Fig. 5. The workspace (the gray area) if the IK solution belong to the same branch than $S_{4}$ (left) or on the same branch than $S_{5}$ (right)

cable has an altitude that is greater than a given threshold $h$ (e.g 0 to avoid having cable on the ground). If $F_{z}<0$ the lowest point of the cable is $B$ whose altitude shall always be larger than $h$. If $F_{z}<0$ then the minimal altitude $h_{m}$ of any point on the cable is

$h_{m}=\frac{E A_{0} F_{x}-\left(L_{0} \mu g-F_{z}\right)^{2} / 2-E A_{0} \sqrt{F_{x}^{2}+\left(F_{z}-\mu g L_{0}\right)^{2}}}{E A_{0} \mu g}$

We may therefore modify our workspace algorithm to take that constraint into account just by defining the extremal poses as the one having one cable $j$ such that either $H_{j}=T^{2}$ or $h_{m}^{j}=h$. This will leads to border arcs that satisfy one of the two constraints.

A second approach is to combine the result of the tension workspace with the one that computes the workspace border when the $L_{0}$ are constrained by $L_{0} \leq L_{\max }$. (which will be presented in [24]) Figure 6 shows both workspaces obtained for $L_{\max }=25$ (W25) and the one for $T=505$.

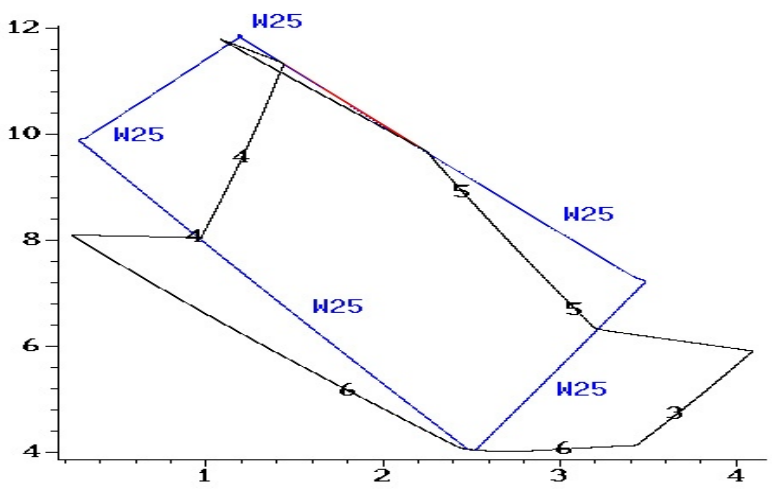

Fig. 6. Workspace for $L_{0} \leq 25$ (W25) and workspace for $T=505$

\section{CDPR WITH MORE THAN 6 CABLES}

\section{A. Kinematic analysis}

If we let $x, y$ be free kinematics has $2+4 n$ unknowns $(x, y$ and the $\left.4 n F_{x}, F_{z}, \alpha, L_{0}\right)$ and $3 n+6$ constraints so that the we have a $n-4$ dimensional variety embedded in the plane. As the dimension of this variety should be 2 this means that the IK is a $n-6$ dimensional variety. As the border of 
the variety is one dimensional this implies that a pose that belongs to the border should also satisfy $n-5$ constraints $H_{j}=T^{2}$. In the remaining section we will assume that $n=8$ so that a point on the border should have 3 cables with their tension at the maximum while the IK is a 2-dimensional variety whose branches coalesce on the border.

\section{B. Finding extremal poses}

At the opposite of the 6-cables case there is an infinite number of solution for the IK but it is still possible to find one particular solution at a given pose $\mathbf{X}_{\mathbf{0}}$ by using interval analysis. Indeed this method allows one to determine small boxes that may include a solution: as soon as such a box is found we consider each pair of $L_{0}$ and fix their values to the mid-value of their ranges. Having fixed these unknowns the IK is becomes a square system that may be solved exactly with interval analysis. From the pose $\mathbf{X}_{\mathbf{0}}$ we move along the $x+, x-, y+, y-$ direction toward a pose $\mathbf{X}_{\mathbf{1}}$ as presented in section III-A except that at each step we consider each pair $(l, m)$ of cables and set their $L_{0}$ to their values at $\mathbf{X}_{\mathbf{0}}$ so that the kinematic equations are square. Using Kantorovitch theorem we are able to determine a step increment $\epsilon$ such that the Newton method will find the solution $\mathbf{X}_{\mathbf{1}}$ of the kinematic system. We then check that for all cables we have $H_{j} \leq T^{2}$. If we found that for one cable $k$ we have $H_{k}>T^{2}$, then we divide $\epsilon$ by 2 and repeat the process. Among all the pairs $(l, m)$ that satisfy $H_{j} \leq T^{2}$ we select as solution the one having the largest $\epsilon$. We then repeat the process using $\mathbf{X}_{\mathbf{1}}$ as new starting point. The process is stopped whenever Newton does not converge for any pair of cable $(l, m)$ (meaning that we are close to a singularity) or when 3 of the $H_{j}$ for $j_{1}, j_{2}, j_{3}$ come very close to $T^{2}$. For finding a point on the border we let the motion variables be free so that we have 33 unknowns while we have the 30 kinematics equations to which we add the 3 constraints $H_{j_{1}}=H_{j_{2}}=H_{j_{3}}=T^{2}$. This square system is solved with Newton to get a pose on the border. The continuation algorithm explained in section III-B may then be used in order to determine border arcs that have now 3 leading cables.

\section{Example}

For this example we use the 8-cables CDPR COGIRO [25]. This robot is a suspended CDPR (i.e. there is no cable pulling the platform downward) with 8 cables, whose $A_{i}, B_{i}$ coordinates are given in table II. Our purpose is to compute a slice of the workspace for $z=2$ while the orientation of the platform is such that the rotation matrix for the platform is the identity. The platform mass is $100 \mathrm{~kg}$ and we set $T=470 \mathrm{~N}$. We choose $(0,0)$ as starting pose from which we deduce 4 poses on the border. After using the end-points of all the arcs we end-up with the workspace presented in figure 7. It may be seen that this workspace is quite complex: a detailed view of the workspace around (-4,2 ) may be seen in figure 8 . It may be seen that the workspace has an outer hull with an interior that is criss-crossed by arcs. These arcs are part of the border for a branch of the

\begin{tabular}{c|c|c|c|c|c|c|}
\multicolumn{1}{c|}{$\mathrm{x}$} & $\mathrm{y}$ & $\mathrm{z}$ & $\mathrm{x}$ & $\mathrm{y}$ & $\mathrm{z}$ \\
\hline \hline-7.175 & -5.244 & 5.462 & -7.316 & -5.1 & 5.47 \\
\hline-7.3 & 5.2 & 5.476 & -7.161 & 5.3 & 5.485 \\
\hline 7.182 & 5.3 & 5.488 & 7.323 & 5.2 & 5.499 \\
\hline & 7.3 & -5.1 & 5.489 & 7.161 & -5.27 & 5.497 \\
$\mathrm{x}$ & $\mathrm{y}$ & $\mathrm{z}$ & $\mathrm{x}$ & $\mathrm{y}$ & $\mathrm{z}$ \\
\hline \hline 0.5 & -0.492 & 0 & -0.5 & 0.35 & 0.997 \\
\hline-0.5032 & -0.2699 & 0 & -0.50321 & 0.49283 & 0 \\
\hline 0.4960 & 0.35562 & 0.99954 & 0.49964 & -0.34028 & 0.99918 \\
\hline 0.5020 & 0.2749 & -0.00062 & -0.50454 & -0.34629 & 0.99752 \\
\hline
\end{tabular}

TABLE II

CoOrdinates of the $A_{i}, B_{i}$ POINTS (In METERS) OF THE COGIRO CDPR

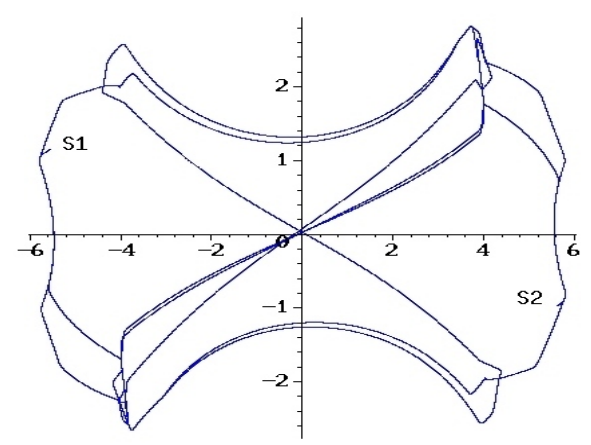

Fig. 7. The workspace border for $T=470$

IK corresponding to a given triplet of cable reaching the maximum. But they may be crossed if another IK solution is chosen. One may also wonder if the workspace with sagging cable is significantly different of the one we will get if we assume ideal cables. As the same algorithm may be used for computing the workspace with ideal cable we present a comparison between both workspace in figure 9. It may be seen that indeed there is large differences between these workspaces.

\section{DETAIL OF THE IMPLEMENTATION}

The workspace algorithm has been implemented in Maple using a multi-precision Newton scheme that allows one to specify the accuracy of the solution and the C++ interval

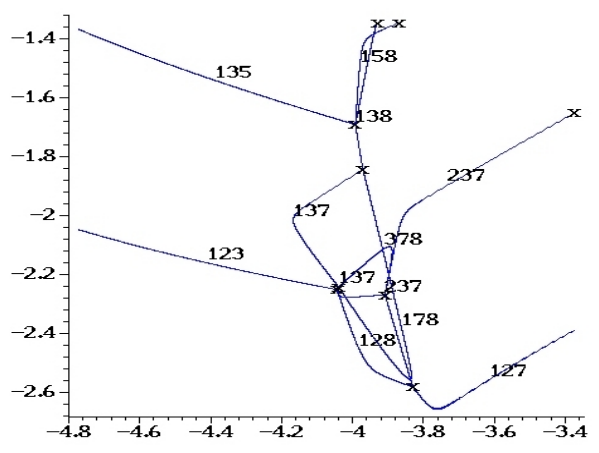

Fig. 8. Detail of the workspace border for $T=470$ around $(-4,-2)$ 


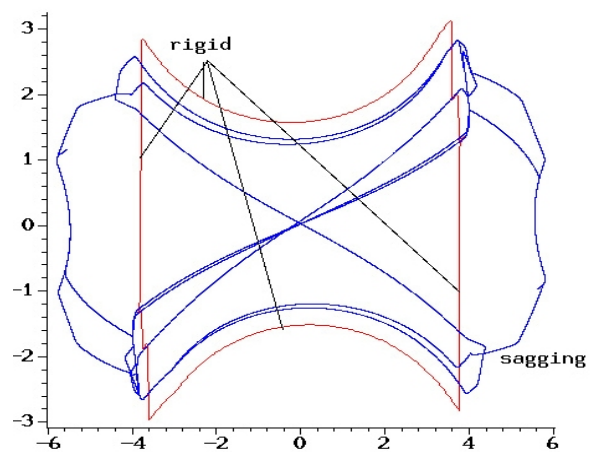

Fig. 9. Comparison between the workspace for ideal and sagging cables for $T=470$

analysis package ALIAS which has a Maple interface so that the workspace algorithm is always run from Maple. For the CDPR example with 6 cables the computation time for the different steps of the algorithm are:

- getting extremal poses from a starting pose: $5 \mathrm{mn}$

- establishing all border arcs not related to a singularity: $15 \mathrm{mn}$

- managing border arcs due to singularity: $30 \mathrm{mn}$

A full C++ implementation should be much faster especially as each of the calculation steps may be implemented in a distributed manner. Note however that in some cases floating point accuracy may not be sufficient to determine correctly border arcs as several of them are in very close proximity. Fortunately the Kantorovitch theorem allows one to detect such a case, enabling to switch to the Maple implementation whenever needed.

\section{CONCLUSION}

Calculating workspace for CDPR with sagging cables is a difficult task because of the complexity of the cable model and because the IK and FK have several solutions. The algorithm proposed in this paper allows one to calculate the workspace border in a reasonable amount of time. Still there are numerous issues that have to be addressed. First of all the algorithm is able to compute the outer hull of the workspace but may miss holes in the inside of the workspace. Second, as seen in the examples, part of the border is obtained not for poses with maximal cable tension(s) but as singular curves and singularities of CDPR with sagging cables has never been addressed in depth. Finally as the computation time is still relatively high one may wonder if it will not be more efficient to start from the workspace border obtained for ideal cables (that is easy and very fast to determine) and use a continuation method on the Young modulus and linear density to slowly modify the shape of the border for getting the workspace border.

\section{REFERENCES}

[1] C. Gosselin, "Determination of the workspace of 6-dof parallel manipulators," ASME J. of Mechanical Design, vol. 112, no. 3, pp. 331-336, September 1990.
[2] J. Albus, R. Bostelman, and N. Dagalakis, "The NIST ROBOCRANE," J. of Robotic Systems, vol. 10, no. 5, pp. 709-724, July 1993.

[3] M. Aref and H. Taghirad, "Geometrical workspace analysis of a cabledriven redundant parallel manipulator: KNTU CDRPM," in IEEE Int. Conf. on Intelligent Robots and Systems (IROS), Nice, France, September, 22-26, 2008, pp. 1958-1963.

[4] X. Diao and O. Ma, "Workspace determination of general 6 d.o.f. cable manipulators," Advanced Robotics, vol. 22, no. 2-3, pp. 261278, 2008.

[5] Q. Duan and X. Duan, "Workspace calculation and quantification calculations of cable-driven parallel robots," Advances in Mechanical Engineering, vol. 2014, 2014.

[6] C. Ferraresi, M. Paoloni, and P. F., "A new methodology for the determination of the workspace of six-dof redundant parallel structures actuated by nine wires," Robotica, vol. 25, no. 1, pp. 113-120, January 2007.

[7] M. Gouttefarde, D. Daney, and J.-P. Merlet, "Interval-analysis based determination of the wrench-feasible workspace of parallel cable-driven robots," IEEE Trans. on Robotics, vol. 27, no. 1, pp. 1-13, February 2011.

[8] J. Jeong, S. Kim, and Y. Kwak, "Kinematics and workspace analysis of a parallel wire mechanism for measuring a robot pose," Mechanism and Machine Theory, vol. 34, no. 6, pp. 825-841, August 1999.

[9] W. Lim et al., "A generic force closure algorithm for cable-driven parallel manipulators," Mechanism and Machine Theory, vol. 46, no. 9, pp. 1265-1275, September 2011.

[10] B. Ouyang and W.-W. Shang, "A new computation method for the force-closure workspace of cable-driven parallel manipulators," Robotica, vol. 33, no. 3, pp. 537-547, March 2015.

[11] J. Pusey et al., "Design and workspace analysis of a 6-6 cablesuspended parallel robot," Mechanism and Machine Theory, vol. 139, no. 7, pp. 761-778, July 2004.

[12] E. Stump and V. Kumar, "Workspaces of cable-actuated parallel manipulators," ASME J. of Mechanical Design, vol. 128, no. 1, pp. 159-167, January 2006.

[13] C. Tavolieri, M. Ceccarelli, and J.-P. Merlet, "A workspace analysis of a fully constrained cable-based parallel manipulator by using interval analysis," in Musme, San Juan, Argentina, April, 8-12, 2008.

[14] R. Verhoeven, "Analysis of the workspace of tendon-based Stewart platforms," Ph.D. dissertation, University of Duisburg-Essen, Duisburg, 2004.

[15] M. Korayem, M. Bamdad, and M. Saadat, "Workspace analysis of cable-suspended robots with elastic cable," in IEEE International Conference on Robotics and Biomimetics, 2007. ROBIO 2007, 2007, pp. 1942-1947.

[16] J.-P. Merlet, "On the workspace of suspended cable-driven parallel robots," in IEEE Int. Conf. on Robotics and Automation, Stockholm, May, 16-20, 2016.

[17] N. Riehl et al., "On the static workspace of large dimension cablesuspended robots with non negligible cable mass," in 34th Annual Mechanisms and Robotics Conference, Montréal, August, 15-18, 2010.

[18] J.-P. Merlet, "Computing cross-sections of the workspace of cabledriven parallel robots with 6 sagging cables," in Computational Kinematics, Poitiers, 2017.

[19] H. M. Irvine, Cable Structures. MIT Press, 1981.

[20] N. Riehl et al., "On the determination of cable characteristics for large dimension cable-driven parallel mechanisms," in IEEE Int. Conf. on Robotics and Automation, Anchorage, May, 3-8, 2010, pp. 47094714

[21] J.-P. Merlet, "A new generic approach for the inverse kinematics of cable-driven parallel robot with 6 deformable cables," in $A R K$, Grasse, June, 27-30, 2016.

[22] R. Tapia, "The Kantorovitch theorem for Newton's method," American Mathematic Monthly, vol. 78, no. 1.ea, pp. 389-392, 1971.

[23] J.-P. Merlet and D. Daney, "A portable, modular parallel wire crane for rescue operations," in IEEE Int. Conf. on Robotics and Automation, Anchorage, May, 3-8, 2010, pp. 2834-2839.

[24] J.-P. Merlet, "Computing cross-sections of the workspace of a cabledriven parallel robot with 6 sagging cables having limited lengths," submitted to ARK 2018.

[25] M. Gouttefarde et al., "Simplified static analysis of large-dimension parallel cable-driven robots," in IEEE Int. Conf. on Robotics and Automation, Saint Paul, May, 14-18, 2012, pp. 2299-2305. 